\title{
Estudio comparativo del manejo del riego por goteo en almen- dro en marco de plantación intensivo y súperintensivo en el Centro Nacional de Tecnología de Regadío (CENTER)
}

\section{Sánchez de Ribera González, Alejandroํㅜㄹ, Cervantes Díaz-Toledo, Alfonso²; Madurga del Cura, Cristina²; Muñoz} Sopeña, Daniel²; Rodríguez Fernández, Diego²; Zazo Salinero, Teresa²

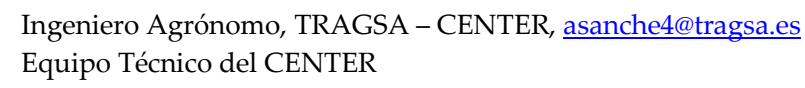

Resumen: El riego por goteo superficial y subterráneo hacen posible que el aporte de agua se haga de un modo preciso, tanto en la cantidad como en la localización del mismo. Se produce un bulbo húmedo que, dependiendo del manejo y del caudal de los emisores, es uniforme a lo largo de ramal, haciendo que mejore la eficiencia de aplicación y, por tanto, la disponibilidad de agua para las plantas.

Desde el Centro Nacional de Tecnología de Regadío se intenta ayudar y concienciar al sector que, para ciertos cultivos como puede ser frutales y leñosos en general, el uso de este tipo de sistemas de riego, así como el uso de las nuevas tecnologías para su manejo, puede ayudar a reducir costes e inputs en el cultivo, manteniendo o incluso aumentando sus rendimientos, además de reducir considerablemente el agua consumida.

El objetivo de este estudio es comparar, mostrar y valorar distintos modelos de sistemas de riego localizado tanto subterráneo como superficial combinado con distintos modos de manejo, bien en modo tradicional o en modo automático, incluyendo sondas y sensores en la toma de decisiones de los riegos, sin que influya el tratamiento agrícola de la planta, que se hará de forma conjunta y por igual, como puede ser el aporte de nutrientes (que se hará por fertirrigación), tratamiento de malas hierbas, enfermedades, poda etc.

En este caso se ha elegido una variedad comercial de almendro específica para implantar 12 parcelas de estudio: 6 parcelas en marco de plantación intensiva de $4 \times 6$ metros y 6 parcelas en marco de plantación superintensiva de $1,25 \times 3,5 \mathrm{~m}$.

Para todas las tuberías emisoras elegidas se realiza una evaluación hidráulica que incluye tanto el coeficiente de uniformidad antes de su instalación y en algunos casos durante su funcionamiento, así como la determinación de la relación presión-caudal suministrada por cada gotero bajo condiciones controladas de temperatura del agua en el Laboratorio de Ensayo de Materiales y Equipos de Riego del CENTER, de acuerdo a la norma UNE-ISO 9261.

Los resultados obtenidos en los distintos años de evolución del cultivo servirán para comparar y valorar los distintos métodos de manejo elegidos y poder mostrar su eficiencia.

Palabras clave: Goteo, Emisor, superficial, subterráneo, almendro, ensayos, CENTER 


\section{Comparative study of the management of drip irrigation in al- mond trees in an intensive and super-intensive plantation framework at the National Center for Irrigation Technology (CENTER).}

Sánchez de Ribera González, Alejandro ${ }^{1}$ Cervantes Díaz-Toledo, Alfonso²; Madurga del Cura, Cristina²; Muñoz Sopeña, Daniel2; Rodríguez Fernández, Diego²; Zazo Salinero, Teresa².

1 Agricultural engineer, TRAGSA - CENTER, asanche4@tragsa.es

2 CENTER Technical team

Abstract: Supply water in a precise way is possible thanks to the surface and underground drip irrigation. Depending on the management and flow of the emitters, a uniform wet bulb is produced along the line of the pipe, improving the efficiency of the application and the availability of water for the plants.

The National Centre for Irrigation Technology (CENTER) tries to help and raise awareness in the sector. In Crops such as fruit trees and woody trees in general, this type of irrigation systems and the new technologies for their management can help to reduce costs and inputs in the crop. It also maintains or even increases productions and reduces considerably the water consumed.

The aim of this study is to compare, show and evaluate different models of localized irrigation systems, underground and surface systems, combined with different management techniques, traditional and automatic mode. Sensors have been also included to make irrigation decisions. The agricultural treatments of the plant are the same for every system and it includes works like applications of nutrients (fertigation mainly), treatments of weeds, diseases, pruning, etc.

The almond variety chosen is a specific commercial variety. The project includes 12 study plots: 6 in an intensive plantation frame of $4 \times 6$ meters and 6 in a super-intensive plantation frame of $1.25 \times 3.5 \mathrm{~m}$.

All emitter pipes used are tested in accordance with the UNE-ISO 9261 standard in the Laboratory of the CENTER. It includes the uniformity coefficient and the pressure-flow relation test supplied by each dripper. All the tests are being performed under controlled conditions of water temperature.

The results obtained in the different years of evolution of the crop will make it possible to compare and evaluate the different management methods chosen and to show their efficiency.

Keywords: Drip, Emitter, superficial, underground, almond tree, tests, CENTER 


\section{Congreso Nacional de Riegos CARTAGENA 2021}

\section{Introducción}

El almendro es uno de los cultivos leñosos que más ha evolucionado en España en los últimos años. Su cultivo ha pasado de ser el cultivo de supervivencia de suelos pobres con producciones de entre 150 y 200 kilos por hectárea $(\mathrm{kg} / \mathrm{ha})$, a las plantaciones intensivas o súper intensivas en regadío que multiplican las producciones, llegando incluso a alcanzar cifras de entre 2500 a $3000 \mathrm{~kg} / \mathrm{ha}$, siendo por lo tanto un cultivo cuyo rendimiento está muy ligado a las dosis de agua aplicadas.

Desde el Centro Nacional de Tecnología de Regadíos, perteneciente a la S.G. de Regadíos, Caminos Naturales e Infraestructuras Rurales del Ministerio de Agricultura, Pesca y Alimentación, siempre se busca fomentar y mejorar las buenas prácticas de aplicación de riegos y apoyar estudios que impliquen comparar distintas formas de aportación hídrica. Este estudio tiene como objetivo comparar, mostrar y valorar distintos modelos de sistemas de riego localizado tanto subterráneo como superficial que a su vez se han combinado con distintos modos de manejo: convencional y automático.

Para evitar incluir más variables que distorsionen esta valoración, se intentará que no influya el tratamiento agrícola de la planta, realizándolo de forma conjunta y por igual, en función de los datos de suelo, y las muestras preliminares de nutrientes, perfiles, $\mathrm{pH}$, etc... Se unificarán tratamientos como: el abonado y la fertilización (que se hará principalmente por fertirrigación), el tratamiento de malas hierbas, el tratamiento de enfermedades, plagas, podas etc...

Este estudio se ha iniciado con la colaboración de varias empresas para la implantación del sistema de riego, las cuales han aportado sus ramales y emisores acorde con sus necesidades. Y una vez que entre en producción el almendro, serán las encargadas de manejar el riego de la parcela correspondiente.

\section{Materiales y métodos}

Para este proyecto se ha establecido una plantación de 2 ha de almendros en regadío subdividida en 12 parcelas, con marcos de plantación y variedad de cultivos acorde a dos modos de plantación que se van a comparar:

- Modo intensivo: consta de seis parcelas de cuatro líneas de cultivo cada una, con un marco de plantación de $4 \times 6$ metros y la variedad de almendro Penta. Cada línea de cultivo tiene un total de 16 árboles por lo que cada parcela de intensivo consta de un total 64 árboles.

- Modo súper intensivo: consta de seis 6 parcelas con seis líneas de cultivo y un marco de plantación de 1,25x 3,5metros y la variedad elegida también es Penta bajo un patrón de ciruelo enanizante "rootpac20" que hace que la planta no se desarrolle tan rápidamente en altura y la formación del seto es más favorable. Cada línea de cultivo tiene un total de 46 árboles por lo que cada parcela de súper intensivo consta de un total de 276 árboles.

Cuando sea época de recolección estos dos modos de plantación también permitirán comparar las formas de recolección y la maquinaria necesaria para ello. 


\section{CXEARY}

\section{Congreso Nacional de Riegos CARTAGENA 2021}

A continuación, se muestra un plano de la situación de la parcela.

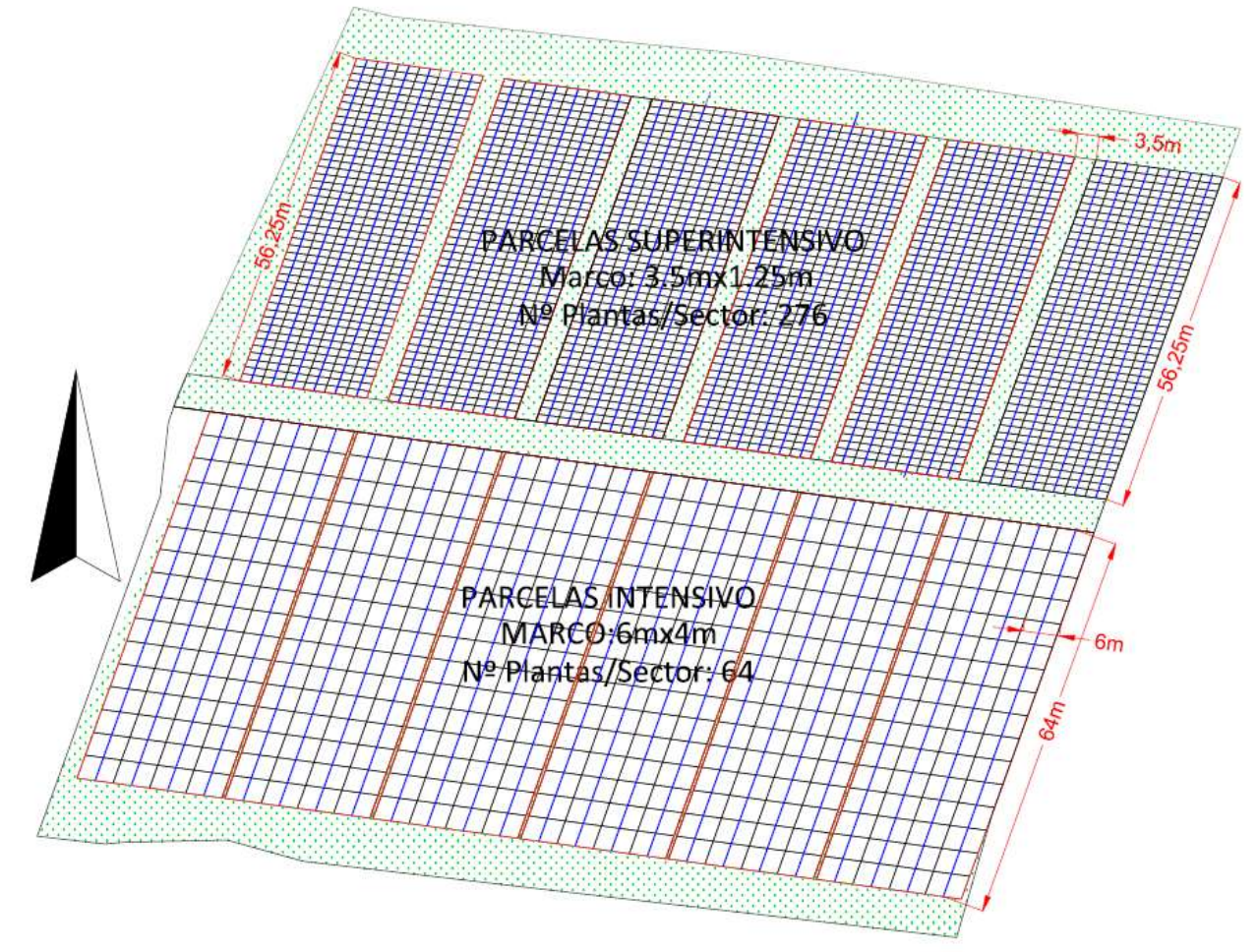

Figura 1. Esquema de distribución de las parcelas.

La variedad elegida para la implantación se ha realizado después de estudiar varias variables como son:

- La ubicación geográfica: el Centro Nacional de Tecnología de Regadíos (CENTER) se encuentra situado en la ribera del río Henares, en el término municipal de San Fernando de Henares, Madrid.

- Los datos agroclimáticos de los últimos 15 años, obtenidos de la estación situada en el propio CENTER perteneciente a la red SIAR.

- El perfil de suelos: aunque se ha elegido para el estudio una parcela de cultivo ya analizada anteriormente se han tomado varios muestreos en las zonas más representativas para poder así, realizar un manejo del riego inicial, lo más eficiente posible.

Tabla 1. Resumen estudio análisis del suelo

\begin{tabular}{ccccccc}
\hline Zona & $\begin{array}{c}\text { Hzte } \\
(\mathbf{c m})\end{array}$ & $\begin{array}{c}\text { Tipo de } \\
\text { suelo }\end{array}$ & PH & \%m.o. & \%CC & $\begin{array}{c}\text { \%Pto } \\
\text { marchitez }\end{array}$ \\
\hline \multirow{2}{*}{$\begin{array}{c}\text { Superin- } \\
\text { tensivo }\end{array}$} & 20 & & 8,5 & 1,0 & 17,0 & 10,4 \\
& 40 & Franco & 8,7 & 0,5 & 18,3 & 10,7 \\
\hline \multirow{3}{*}{ Intensivo } & 60 & & 8,7 & 0,4 & & \\
& 20 & Franco & 8,5 & 1,0 & 22,7 & 12,8 \\
& 40 & limoso & 8,5 & 0,5 & 20,2 & 9,8 \\
\hline
\end{tabular}


Con los estudios realizados, aunque predomina el suelo franco, se ha detectado una zona un poco más arenosa (60-70\%) sobre todo en horizontes más profundos que hay que tener en cuenta para realizar un manejo del riego lo más adecuado posible. Para estas zonas concretas se realiza un seguimiento especial.

Los datos de porcentaje de materia orgánica en el suelo indican el valor del aporte inicial necesario para la implantación del cultivo, como así se ha hecho.

Los valores de $\mathrm{pH}$ nos sirven para conocer el comportamiento del suelo y ver la incidencia que tiene en la planta y las raíces, ya que de ello va a depender que se asimilen mejor o peor los nutrientes.

Los valores de capacidad de campo y punto de marchitez dan una aproximación de la humedad mínima que debe tener el suelo para que, sin estar saturado, siempre tenga agua disponible la planta para su buen desarrollo durante los periodos de crecimiento y las distintas fases (brotación, cuajado y crecimiento de los frutos).

- Nutrientes en el suelo: también se ha realizado un muestreo en varios puntos para conocer el estado inicial, determinar las carencias del suelo en cuanto a macro y micro nutrientes y poder realizar los aportes necesarios. Los resultados más representativos se muestran a continuación:

Tabla 2. Resumen muestras nutrientes en el suelo

\begin{tabular}{ccc}
\hline Nutriente & M1 (mg/kg suelo) & M2(mg/kg suelo) \\
\hline $\mathrm{N}$ & 1,38 & 13,34 \\
$\mathrm{~N}-\mathrm{NO}_{3}$ & 6,1 & 59,07 \\
$\mathrm{P}$ & 17,63 & 25,22 \\
$\mathrm{~K}$ & 577,69 & 892,43 \\
$\mathrm{Ca}$ & 4794,82 & 5501,99 \\
$\mathrm{Mg}$ & 384,46 & 555,78 \\
$\mathrm{Na}$ & 65,54 & 90,34 \\
\hline
\end{tabular}

Con todos estos datos analizados, se decidió que la variedad debía presentar una época de floración muy tardía, para poder eludir las posibles heladas primaverales de la zona. Es por eso que tras valorar las diferentes posibilidades, se ha elegido como variedad principal Penta.

Desde el principio de la plantación que se realizó en Junio/Julio del año 2020 se ha hecho un seguimiento tanto a la planta como al riego. 
Respecto al riego en esta campaña se ha hecho hincapié en el buen desarrollo de la planta, ya con todos los emisores implantados desde el mes de junio de 2021.

Las distintas parcelas estudiadas se corresponden con las siguiente distribución del riego:

Tabla 3. Resumen del riego instalado Caudal $(1 / \mathrm{h})$ y separación entre emisores $(\mathrm{m})$ en cada parcela de ensayo.

\begin{tabular}{|c|c|c|c|}
\hline $\begin{array}{c}\text { № } \\
\text { Parcela }\end{array}$ & $\begin{array}{l}\text { Intensivo } 4 \text { filas } \\
6 \times 4 \mathrm{~m}\end{array}$ & $\begin{array}{c}\text { Súperintensivo } 6 \text { filas } \\
\text { de } 3,5 \times 1,25 \mathrm{~m}\end{array}$ & $\begin{array}{c}\mathrm{N}^{\mathrm{o}} \\
\text { Parcela }\end{array}$ \\
\hline 11 & $\begin{array}{c}\text { Qe: 1,6 1/h; Se: 0,6m } \\
\text { Riego superficial }\end{array}$ & $\begin{array}{l}\text { Qe: } 2 \text { 1/h; Se: 0,5m } \\
\text { Riego enterrado }\end{array}$ & 12 \\
\hline 9 & $\begin{array}{l}\text { Qe: } 1,6 \text { 1/h; Se: } 0,6 \mathrm{~m} \\
\text { Riego enterrado }\end{array}$ & $\begin{array}{l}\text { Qe: 1,6 1/h Se: 0,6m } \\
\text { Riego superficial }\end{array}$ & 10 \\
\hline 7 & $\begin{array}{l}\text { Qe: } 1,5 \text { 1/h; Se: } 0,6 \mathrm{~m} \\
\text { Riego enterrado }\end{array}$ & $\begin{array}{c}\text { Qe: } 1,5 \text { 1/h Se: } 0,6 \mathrm{~m} \\
\text { Riego enterrado }\end{array}$ & 8 \\
\hline 5 & $\begin{array}{l}\text { Qe: 1,5 l/h; Se: 0,6 } \\
\text { Riego enterrado m }\end{array}$ & $\begin{array}{c}\text { Qe: } 1,5 \text { 1/h Se: } 0,6 \mathrm{~m} \\
\text { Riego enterrado }\end{array}$ & 6 \\
\hline 3 & $\begin{array}{l}\text { Qe: } 2 \text { 1/h; Se: 0,75m } \\
\text { Enterrado2 }{ }^{\circ} \text { año }\end{array}$ & $\begin{array}{c}\text { Qe: } 1,6 \text { 1/h Se: } 0,6 \mathrm{~m} \\
\text { Riego enterrado } 2^{\circ} \text { año }\end{array}$ & 4 \\
\hline 1 & $\begin{array}{l}\text { Qe: 1,6 l/h; Se: 0,5m } \\
\text { Riego superficial }\end{array}$ & $\begin{array}{c}\text { Qe: 1,6 1/h Se: 0,5m } \\
\text { Riego superficial }\end{array}$ & 2 \\
\hline
\end{tabular}

Desde el principio, se ha ido registrando los siguientes datos de cada parcela que ayudaran a evaluar los distintos sistemas implantados:

- Tubería instalada en cada sector. Si se ha enterrado o no y desde cuándo.

- Control de los riegos y lectura de contadores: dentro de la programación de riegos, controlar y revisar consumos por parcela. Detección de fugas y posibles emisores obturados.

- Presión de riego. Se regula a la entrada de cada parcela, mediante una válvula hidráulica reductora, la presión de riego recomendada para cada tipo de tubería emisora instalada.

- Registro de precipitaciones: se lleva un histórico de las precipitaciones que se han producido y la efectividad de las mismas, en las distintas épocas.

- Lectura de sondas de humedad y otros sensores de las parcelas que están instalados.

- Datos de fertirrigación: se lleva registro de los aportes de abonado que se han realizado en cada momento para controlar el aporte de nutrientes. Como se ha comentado anteriormente este aporte es común a todas las parcelas para que no sea un condicionante a la hora de comparar manejos de riego. Estos aportes se han calculado basándose en estudios, publicaciones [1-2] y asesoramiento de técnicos expertos en la materia, que, junto con los análisis realizados, han recomendado los aportes asignados. Esto se continuará haciendo durante los años sucesivos. 


\section{Congreso Nacional de Riegos CARTAGENA 2021}

Paralelamente se va a evaluar distintos sistemas de manejo con distintas colaboraciones:

1. El manejo tradicional o convencional, que, mediante los modelos estadísticos y métodos empíricos, los coeficientes de cultivo (FAO), mezclado con los datos climáticos y de suelo, establecen las dosis de riego.

2. El manejo en modo automático en el que se tiene en cuenta todo lo anterior, pero a su vez, se programa el riego incluyendo los datos suministrados principalmente por sondas de humedad y otros tipos de sensores.

Para ayudar a ser más exhaustivos, todas las tuberías emisoras y los emisores instalados en campo se están ensayando en el banco de emisores del Laboratorio Central para Ensayo de Materiales y Equipos de Riego del CENTER, de forma que se tenga perfectamente determinado el coeficiente de uniformidad y la curva caudal presión (Q-P). Ver ejemplo Fig.5.

Cada parcela de riego está conectada por un sistema de telecontrol que aparte de abrir y cerrar la válvula hidráulica reductora, acumula la lectura de pulsos del contador, da información de la presión en la tubería principal y acumula el gasto total de las 12 parcelas.

También se tendrán en cuenta, cuando la planta empiece a producir almendra, otros aspectos importantes como pueden ser:

- Fecha de entrada en producción

- Rendimientos de producción por ha dependiendo de las dosis aplicadas.

- Otros aspectos como, el coste de mano de obra según los costes por hora y ha, el personal requerido de mantenimiento, costes de recolección y tiempo invertido, etc...

\section{Resultados y discusión}

Después de un año de seguimiento, se lleva registro de todos los consumos, presiones y gastos por parcela descritos anteriormente. A continuación, se muestran los consumos por parcela registrados:

Tabla 4. Resumen del gasto medio registrado por parcela $(1 / \mathrm{h})$

\begin{tabular}{cccc}
\hline Parcela & Gato (1/h) Intensivo & Gasto (1/h) Súperinten. & Parcela \\
\hline 11 & 1365 & 2025 & 12 \\
9 & 1365 & 1350 & 10 \\
7 & 1280 & 1687,5 & 8 \\
5 & 1280 & 1688 & 6 \\
3 & 1365 & 900 & 4 \\
1 & 1638 & 2160 & 2 \\
\hline
\end{tabular}




\section{Congreso Nacional de Riegos CARTAGENA 2021}

También se tiene registrados resultados de:

- Datos de los sensores y las sondas de humedad instaladas. Los resultados obtenidos permiten conocer el estado del terreno y ayuda a la toma de decisiones tanto a nivel instantáneo, como a nivel global de campaña, así como a estudiar la evolución y el comportamiento de la parcela. Por ejemplo:

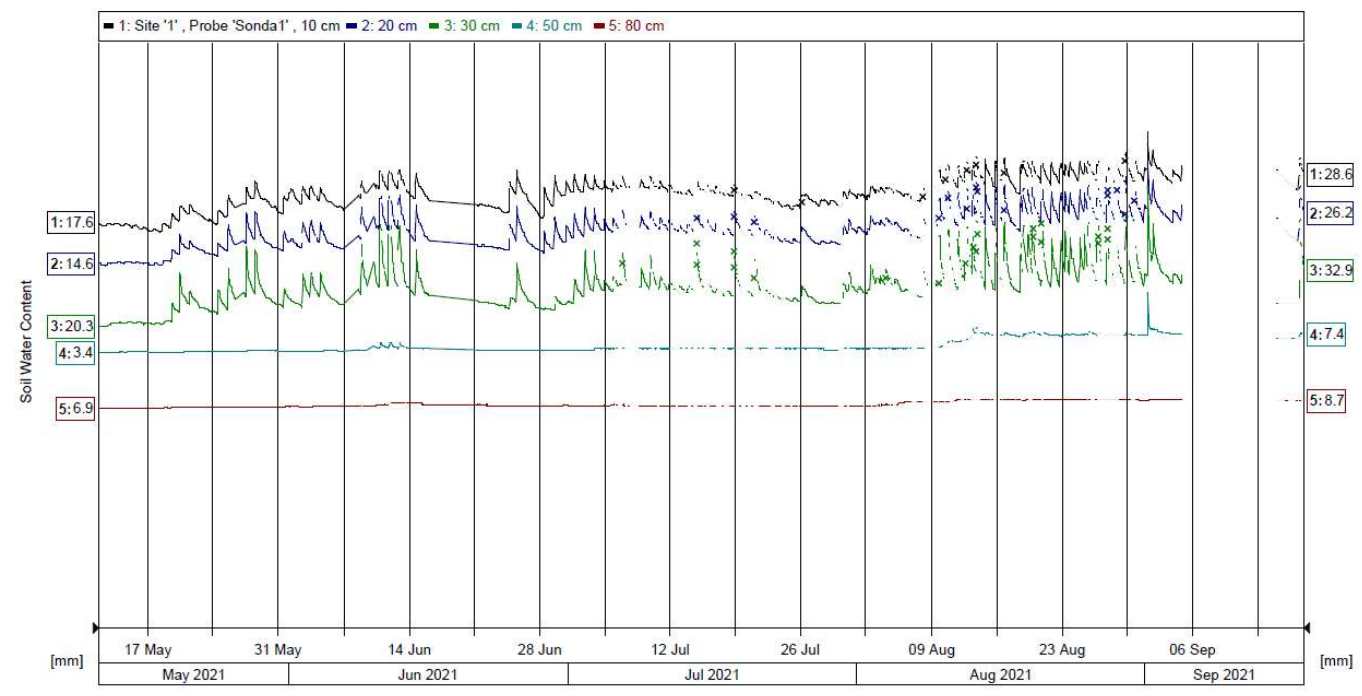

Figura 2. Visualización de la campaña de riego visto desde una sonda de humedad.

- Perímetro del tallo de árboles elegidos para el muestreo de cada parcela de ensayo.

- Altura de la planta, aunque se han realizado varias podas de formación.

- Aspecto general en cuanto a vigor color y estado general, sobre todo para localizar posibles plagas y enfermedades, y realizar las reposiciones de marras necesarias.

A continuación se muestra la evolución de las parcelas desde su plantación en junio de 2020 hasta septiembre de 2021.

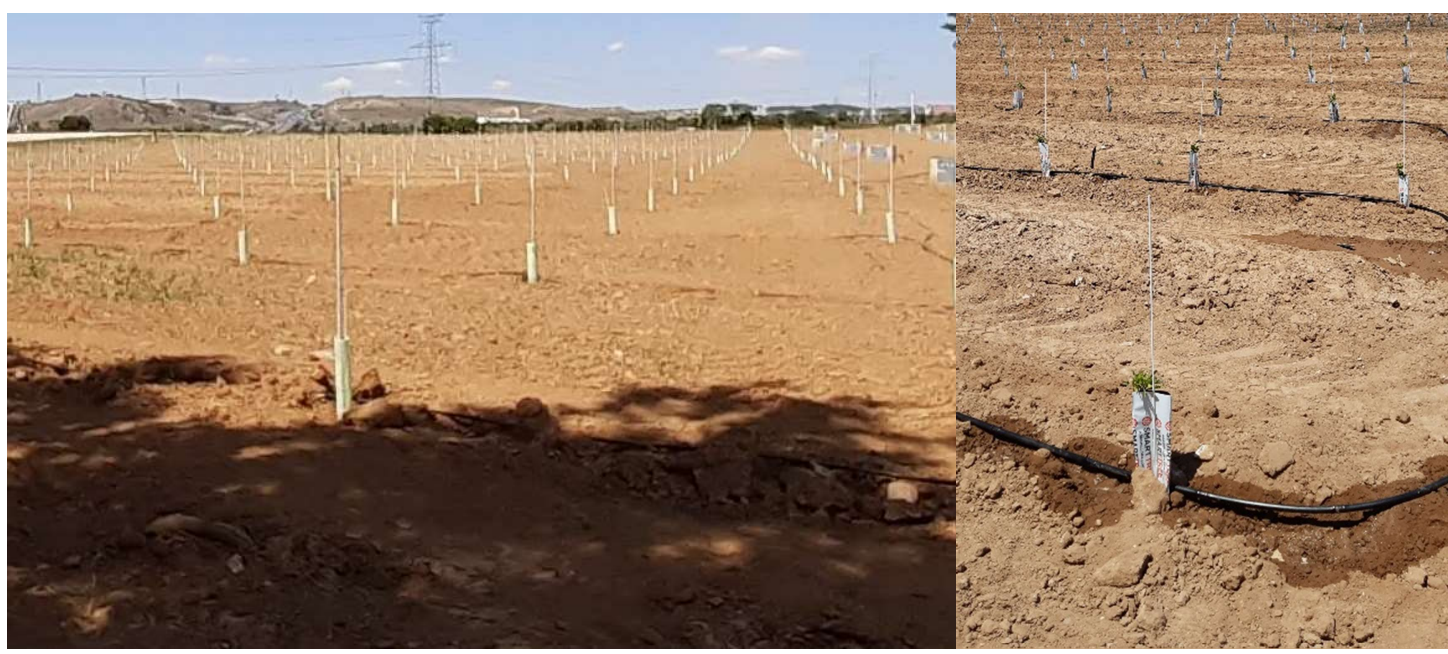

Figura 3. Estado del cultivo despues de su plantación. 


\section{CXeAro}

CARTAGENA 2021

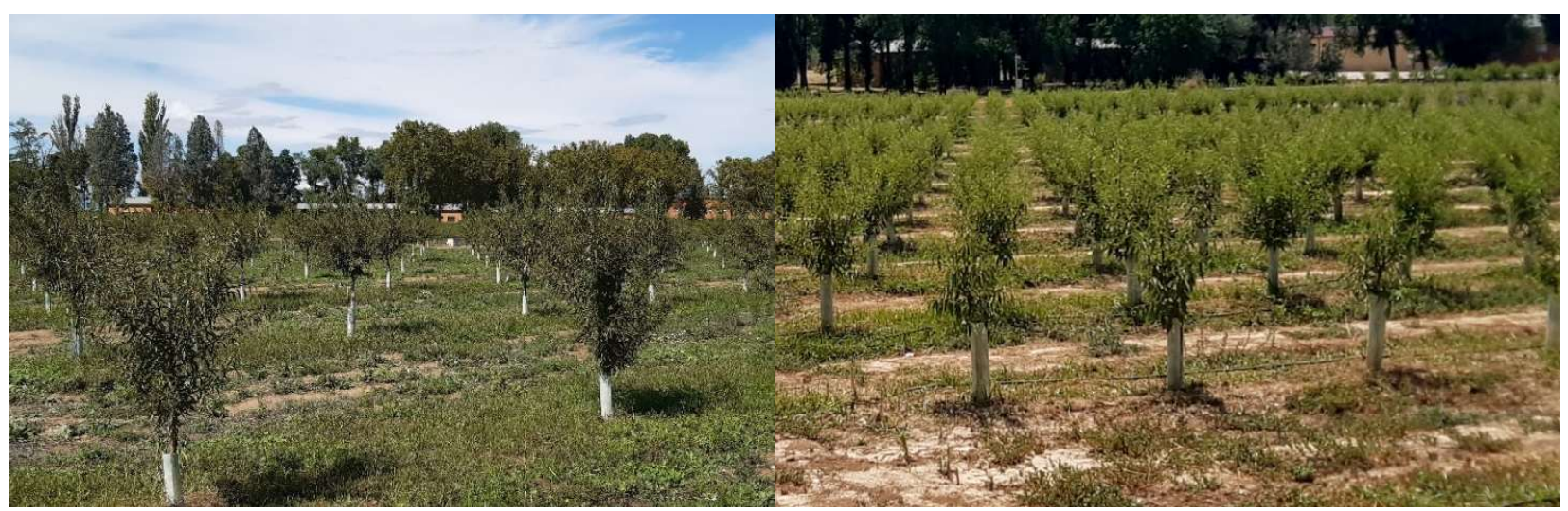

Figura 4. Estado del cultivo en la actualidad.

- Datos de ensayo de los emisores, a continuación, se muestra un ejemplo de la curva caudal-presión de uno de los emisores instalados, perteneciente a una tubería emisora compensante.

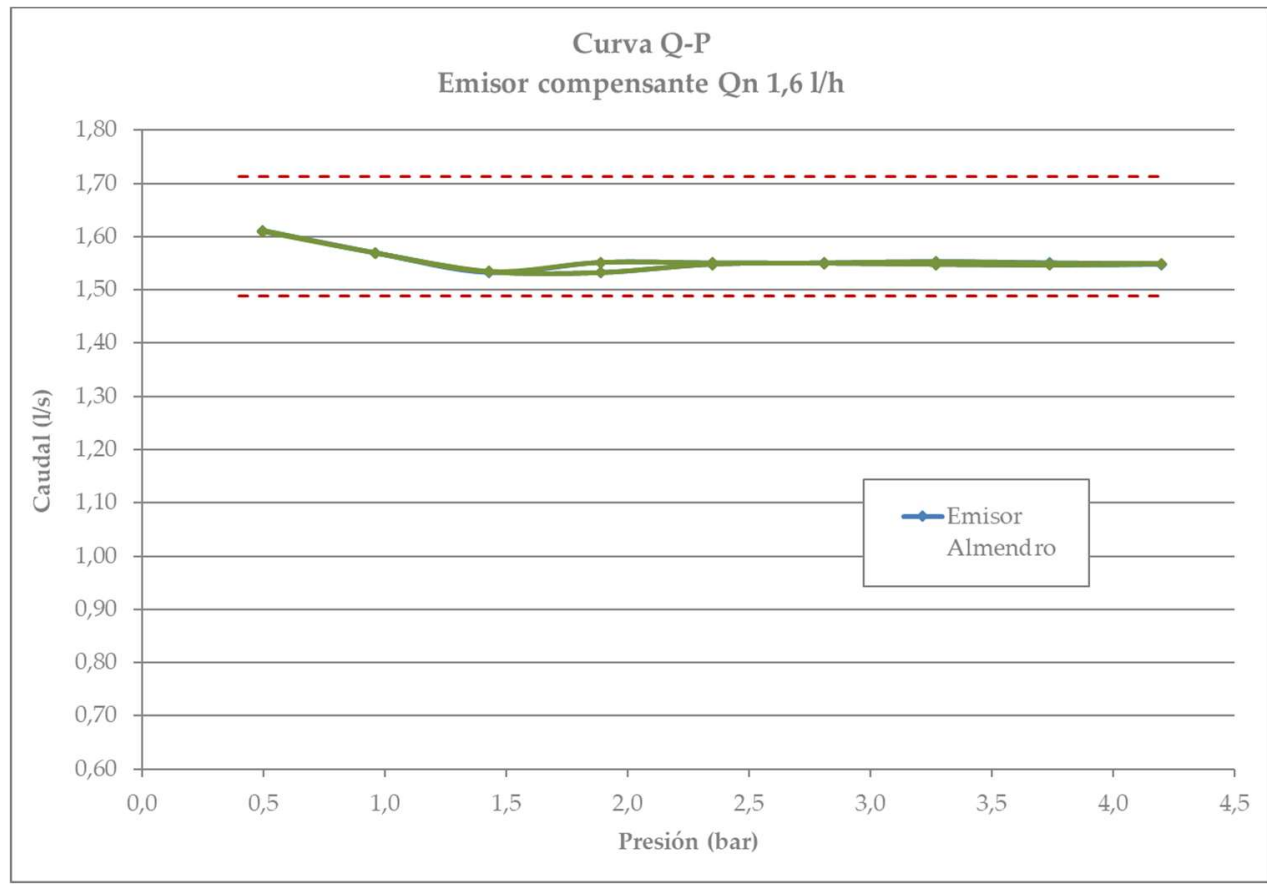

Figura 5. Curva caudal-presión emisor compensante instalado en campo rango de compensación (0.4-3.5 bar). 


\section{Conclusiones}

Aunque se está en el arranque del proyecto y solo se lleva una campaña completa de riego, se empiezan a ver algunas cuestiones importantes de cara a la implantación y evolución de un cultivo de este tipo, como puede ser las siguientes:

1. Los estudios previos de parcela: ubicación, suelo, nutrientes, etc... se hacen muy necesarios para la implantación correcta de un cultivo como el almendro, ya que condicionan no solo la variedad sino también el sistema de riego y su manejo.

2. El control de malas hierbas, en los estados iniciales, es una tarea complicada y difícil de realizar, que, aunque no es objeto de este proyecto, se hace difícil no reflejarlo en las conclusiones debido a la labor inicial que supone. Se recomienda realizar un control exhaustivo para evitar su descontrol, bien mezclando herbicidas que no dañen el almendro en su fase inicial, cosa que se hace complicada en su manejo y efectividad final, bien desbrozado de manera constante cuando sea necesario, también teniendo cuidado con no dañar la planta, o bien mezclando eficazmente las dos anteriores.

3. La buena proyección y dimensionado de la plantación evita bastantes gastos posteriores que surgen en la puesta en marcha de la instalación, y que pueden afectar directamente al desarrollo homogéneo de las plantas.

4. En el caso del almendro, un buen manejo de riego inicial se hace necesario para un buen desarrollo de la planta, de forma que muchos factores de este manejo influyen, como puede ser: el enterrado o no de la tubería emisora desde el inicio, la limpieza recurrente de sectores después de alguna rotura o desajuste de la tubería emisora, la colocación de purgadores para mejorar el funcionamiento, etc...

5. La falta de algunos nutrientes, la radiación solar (zonas de sombra en algunos puntos) y errores en el manejo del riego, inciden de manera directa en el desarrollo homogéneo del cultivo.

Es por esto que un buen manejo y la elección del sistema de riego correcto desde el inicio, es importante y necesario para hacer prosperar el cultivo y facilitar las cosas al usuario del sistema de riego.

\section{Referencias}

1. Guía de gestión integrada de plagas Almendro Ministerio de Agricultura, Pesca y Alimentación.

2. Guía práctica fertilización racional de cultivos en España. parte II, Ministerio de Medio Ambiente y Medio Rural y Marino 